\title{
Identification of binary and multiple systems in TGAS using the Virtual Observatory
}

\author{
F. Jiménez-Esteban ${ }^{1,2,3}$ and E. Solano ${ }^{1,2}$ \\ ${ }^{1}$ Centro de Astrobiología (INTA-CSIC), Madrid, Spain \\ email: fran.jimenez-esteban@cab.inta-csic.es \\ ${ }^{2}$ Spanish Virtual Observatory \\ ${ }^{3}$ Suffolk University
}

\begin{abstract}
Binary and multiple stars have long provided an effective method of testing stellar formation and evolution theories. In particular, wide binary systems with separations $>20,000$ au are particularly challenging as their physical separations are beyond the typical size of a collapsing cloud core $(5,000-10,000 \mathrm{au})$. We present here a preliminary work in which we make use of the TGAS catalogue and Virtual Observatory tools and services (Aladin, TOPCAT, STILTS, VOSA, VizieR) to identify binary and multiple star candidate systems. The catalogue will be available from the Spanish VO portal (http://svo.cab.inta-csic.es) in the coming months.
\end{abstract}

Keywords. Astronomical data bases, catalogues, virtual observatory tools, parallaxes, proper motions, binaries: visual.

\section{Search of co-moving candidates}

We selected TGAS (Gaia Collaboration et al. 2016) sources with positive parallaxes and errors $<10 \%$ in both proper motion components and parallaxes (250,466 sources). Co-moving systems were defined as any group of sources with the same parallaxes and proper motions within 3 times the sum of the individual errors $(\sim 150,000$ candidate pairs). We set a conservative limit of $500,000 \mathrm{au}(\sim 2.5 \mathrm{pc})$ in the projected sky separation to discard systems whose components are too far to be physically bound. 15,645 sources grouped in almost 7,000 systems remained.

\section{Radial velocity confirmation}

We searched radial velocity (RV) data at RAVE-DR5 (Kunder et al. 2017) and LAMOSTDR2 (Luo et al. 2015) catalogues, both available at Vizier. RVs were found for 2,243 sources. The components of 339 systems ( 5\%) showed the same RV within 3 times the sum of the individual errors. 459 sources were rejected because of their discrepant RV values. This left 15,186 sources in 6,703 systems (339 confirmed and 6364 candidate).

\section{Binding Energies}

We used the Gaia-2MASS $\left(\mathrm{G}-\mathrm{K}_{s}\right)$ color and the absolute magnitude $\mathrm{M}_{G}$ obtained using the TGAS distance to place our candidate systems in a H-R diagram (Fig. 1). According to their position 804 objects $(\sim 5 \%)$ were classified as subgiants or giants and 13,400 as dwarfs.

Spectroscopic determinations of the effective temperatures $\left(\mathrm{T}_{\text {eff }}\right)$ of 1,972 stars were obtained from RAVE-DR5, LAMOST-DR2, and PASTEL (Soubiran et al. 2016). For the rest of objects, $T_{\text {eff }}$ were estimated using VOSA (Bayo et al. 2008). (Fig. 2). 


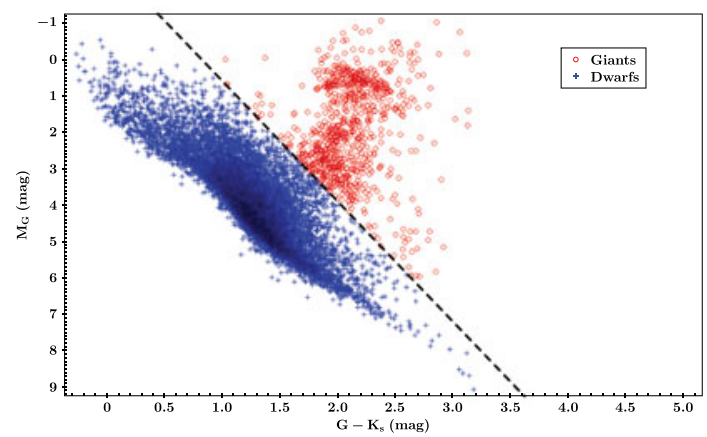

Figure 1. H-R diagram showing the location of our candidate systems. Dashed line separates dwarfs (below) from giants (above).
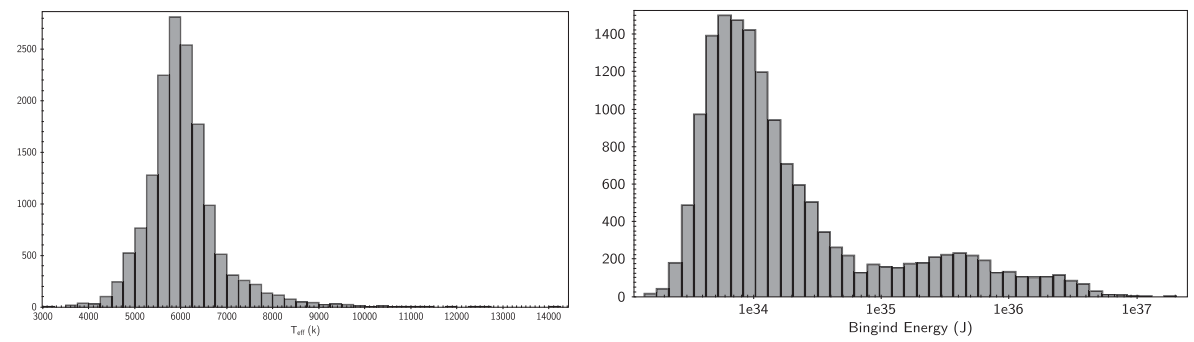

Figure 2. Left: $\mathrm{T}_{\text {eff }}$ distribution. The mayority of our objects show effective temperaturs typical of F-G spectral types; Right: Binding energy distribution. The bi-modal shape reported in Dhital et al. (2010) is also found here.

Stellar masses were derived by interpolating $\mathrm{T}_{\text {eff }}$ in the tables B1 and B2 (Gray 2008) using the dwarf/giant information. Masses and projected physical separation were used to estimate the binding energy $\mathrm{U}=-\mathrm{GM}_{1} \mathrm{M}_{2} / \mathrm{r}$ (Fig. 2). Following Dhital et al. (2010), we set a lower limit for the binding energy of $10^{33} \mathrm{~J}$.

An analysis of the distribution of binding energies and the identification of interesting systems (weakly bound, FGK-M pairs,...) is currently on-going.

\section{Acknowledgements}

This work has made use of data from the European Space Agency (ESA) mission Gaia (https://www.cosmos.esa.int/gaia), processed by the Gaia Data Processing and Analysis Consortium (DPAC, https://www.cosmos.esa.int/web/gaia/dpac/consortium). Funding for the DPAC has been provided by national institutions, in particular the institutions participating in the Gaia Multilateral Agreement.

\section{References}

Bayo, A., Rodrigo, C., Barrado Y Navascués, D., et al. 2008, A $\& A$ A, 492, 277

Dhital, S., West, A. A., Stassun, K. G., \& Bochanski, J. J. 2010, AJ, 139, 2566

Gaia Collaboration et al. 2016, A\&A, 595, A2

Gray, D. F. 2008, The Observation and Analysis of Stellar Photospheres, by David F. Gray, Cambridge, UK: Cambridge University Press

Kunder, A., Kordopatis, G., Steinmetz, M., et al. 2017, AJ, 153, 75

Luo, A.-L., Zhao, Y.-H., Zhao, G., et al. 2015, Research in Astronomy and Astrophysics, 15, 1095

Soubiran, C., Le Campion, J.-F., Brouillet, N., \& Chemin, L. 2016, A\&SA, 591, A118 